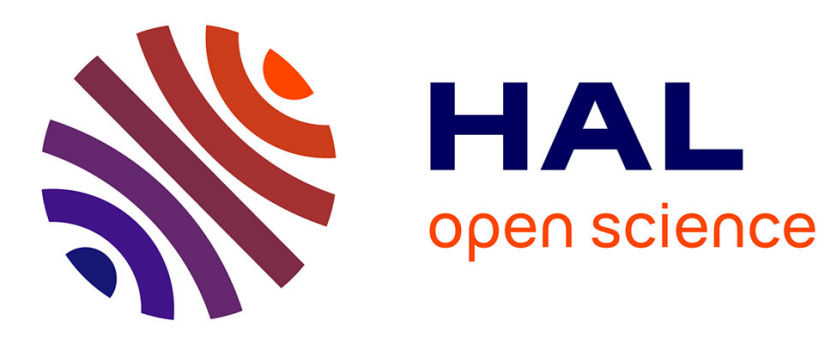

\title{
Revealing modes from controlling an off-optical axis conical diffraction laser
}

\author{
Alain Brenier
}

\section{To cite this version:}

Alain Brenier. Revealing modes from controlling an off-optical axis conical diffraction laser. Laser Physics, 2017, 27 (10), pp.105001. 10.1088/1555-6611/aa7835 . hal-02379628v2

\section{HAL Id: hal-02379628 \\ https://hal.science/hal-02379628v2}

Submitted on 30 Jan 2020

HAL is a multi-disciplinary open access archive for the deposit and dissemination of scientific research documents, whether they are published or not. The documents may come from teaching and research institutions in France or abroad, or from public or private research centers.
L'archive ouverte pluridisciplinaire HAL, est destinée au dépôt et à la diffusion de documents scientifiques de niveau recherche, publiés ou non, émanant des établissements d'enseignement et de recherche français ou étrangers, des laboratoires publics ou privés. 


\title{
Revealing modes from controlling an off-optical axis conical diffraction laser
}

\author{
Alain Brenier \\ Institut Lumière Matière, UMR5306 Université Lyon 1-CNRS, Université de Lyon 69622 Villeurbanne Cedex, France \\ alain.brenier@univ-lyon1.fr
}

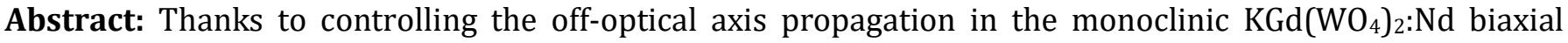
crystal used as a laser gain medium, we exhibited remarkable crescent laser modes and mode switching, demonstrating the preservation of the conical diffraction. We revealed an optical singularity in addition to the optical axis: the directions of polarization abrupt change of the highest emission cross-section mode, which leads to un-polarized lasing. The key explanation is a severe mode selection due to the threshold behavior of lasing. This is confirmed by a theoretical model taking into account a two-axes crystal rotation and including the conical diffraction behavior. The rotation around the two-fold b crystallographic axis of the frame which makes diagonal the imaginary part of the dielectric susceptibility tensor is exhibited.
\end{abstract}

PACS: 42.55.Xi Diode-pumped lasers; 42.25.Ja Polarization

Keywords: biaxial laser crystal

\section{Introduction}

Singularities in physics, especially in wave propagation or optics, have attracted much attention because they lead to intriguing and beautiful observations [1]. Light is constituted of electric and magnetic vector-fields, so it has a polarization which has an essential role when it propagates inside a birefringent material, natural or artificial, with wanted or unwanted effects eventually depending on its control [2]. Propagation inside an anisotropic medium can lead to polarization singularities. Among them we find the famous conical diffraction in biaxial crystals discovered theoretically by Hamilton and experimentally by Lloyd in the nineteenth 
century. This phenomenon occurring during propagation along the optical axis is multi-facetted and was studied all along the twentieth century [3-5] and up to nowadays [6], including applications: polarization demultiplexing and multiplexing [7] for free-space optical communication, optical trapping of micro-spheres [8], enhanced resolution microscopy by sub-wavelength localization [9-10], two-photon polymerization [11], and polarimetry [12-13]. Laser emission from a biaxial doped crystal oriented along its optical axis has been performed [14-18] during the last decade and a link to crystal optics established [19].

On the other hand, the absorption anisotropy splits an optical axis in two new singular ones, each propagating unchanged a right or left circularly polarized light. Launching the inverse circular polarization, i. e. left or right respectively, leads to propagation of the singular Voigt wave [20-21] which is characterized by a linear dependence of its amplitude superimposed to the usual exponential one. Its existence in a medium depends on parameters pointed out in [22]. This wave could find applications in optical sensing [23] for example from a porous biaxial dielectric, and is extensively studied in metamaterials [24].

Beam propagation exactly aligned with the optical axis is desirable in conical diffraction experiments where the transparent anisotropic medium is passive: only this direction is singular and misalignment should lead to trivial degraded patterns and progressively to common double refraction. This was the protocol since the early times of Lloyd demonstration (1832). To the contrary and breaking here this rule we handle a laser gain medium: the monoclinic $\mathrm{KGd}\left(\mathrm{WO}_{4}\right)_{2}: \mathrm{Nd}(\mathrm{KGW}: \mathrm{Nd})$ biaxial crystal, and we exploit a controlled off-optical axis propagation to exhibit remarkable transverse laser modes and mode switching. We reveal for the first time (at our knowledge) that some optical singularities are able to deeply modify lasing.

\section{Experimental set-up}

The experimental laser design is represented in Fig. 1, including an optical axis-oriented KGW:Nd crystal whose optical axis can be slightly rotated by respect to the cavity axis. The laser cavity is plan-concave and pumped by a Limo fibre coupled laser diode $(0.22 \mathrm{NA}$ and $200 \mu \mathrm{m}$ diameter $)$ at $810 \mathrm{~nm}$ through the concave mirror (5 cm radius curvature, HT@810 nm, HR>99.5\% @1068 nm). The pumping was limited to $480 \mathrm{~mW}$ to minimize the thermal fluctuations. A dichroic beamsplitter (HT@810 nm and HR@1068 nm) is inserted before the input mirror in order that the $1068 \mathrm{~nm}$ leaks are directed towards a polarizer and a power-meter (not represented in Fig. 1). The laser polarization and power can also be measured of course through the plane output mirror which has 5\% transmission. The cavity axis defines a fixed z" axis used as a reference. The laser KGW:Nd crystal has 3\% Nd doping, it has $3.15 \mathrm{~mm}$ thickness and it is cut perpendicular to the $\mathrm{z}^{\prime}$ optical axis as shown in Fig. 2 (a). It is inserted in a water cooled copper holder equipped with three orthogonal axes (one longitudinal and two transverse) for rotation around the optical 


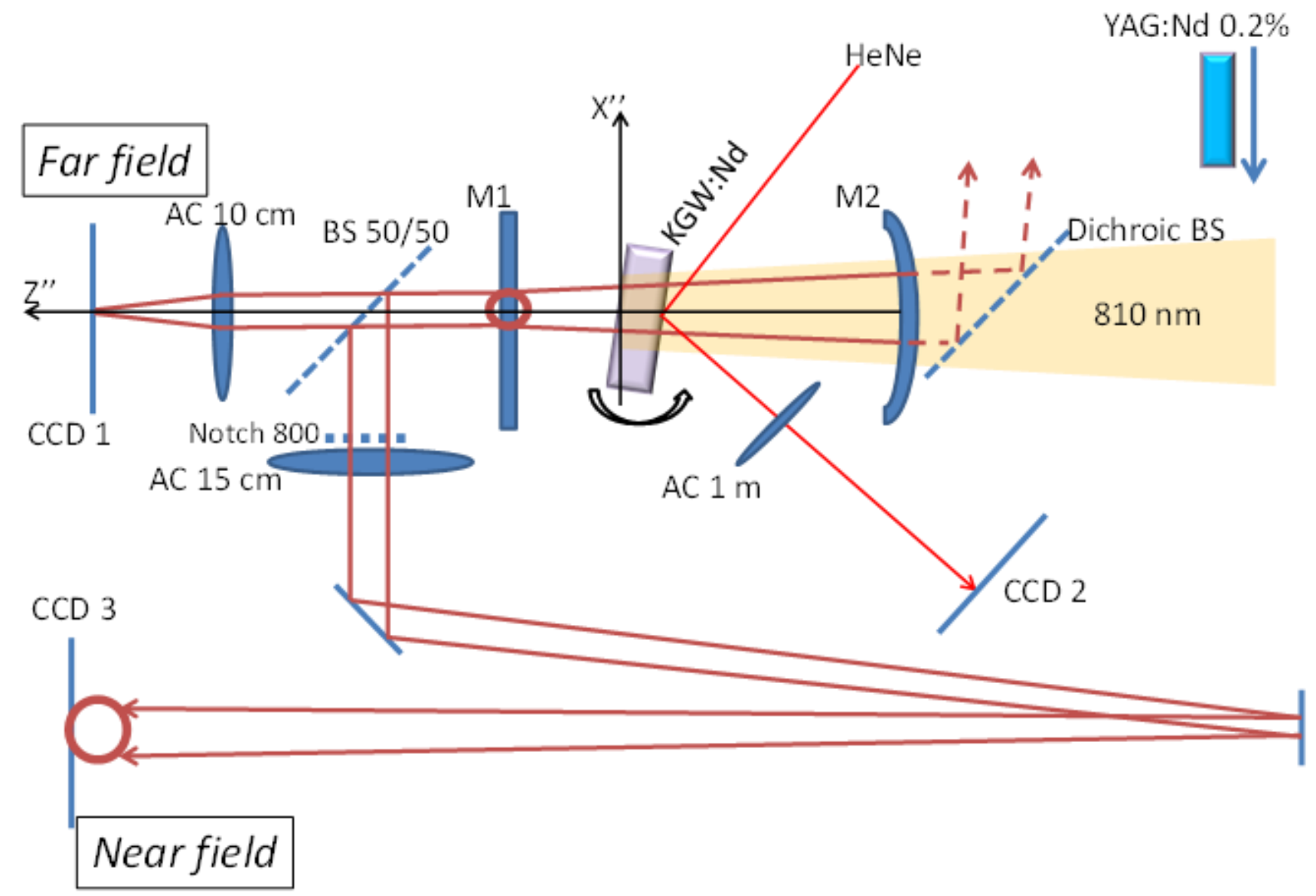

Fig. 1 Experimental set-up of the conical diffraction laser with the control of the laser crystal orientation.

axis. The orientation of the crystal was verified by conoscopy. For this preliminary step we used the fluorescence of a Nd-doped YAG crystal located in the pump path. The fluorescence was focused through a vertical polarizer on the crystal (after removing the two cavity mirrors) and detected through a horizontal polarizer and a $1064 \mathrm{~nm}$ interference filter on the screen of a CCD camera (labelled CCD1) located in the focal plane of a $10 \mathrm{~cm}$ focal length doublet. The optical axis z' and the $\mathrm{x}=\mathrm{Np} / \mathrm{z}=\mathrm{Ng}$ principal plane were clearly identified, allowing the alignment of z' with the cavity optical axis z" as the starting configuration of the las er experiment. The longitudinal axis rotation was used to orient horizontally the $x=N_{p} / y=N_{g}$ principal plane before starting the experiment, then this axis was kept fixed. To the contrary the two horizontal and vertical axis actuators were adjusted all along the experiment. A key-point is that during laser operation the two $\theta_{\mathrm{V}}$ and $\theta_{\mathrm{H}}$ rotation angles were explored and captured on the screen of a CCD camera (labelled CCD2) located in the focal plane of a $1 \mathrm{~m}$ focal length doublet from the reflection of a beam originating from a Helium-Neon laser. 
The intensity of the laser mode on the output mirror (near field) is imaged with 20 times magnification on the camera screen of a third CCD camera (labelled CCD3) with a $15 \mathrm{~cm}$ focal length doublet through a 50/50 beam splitter.

\section{Experimental results}
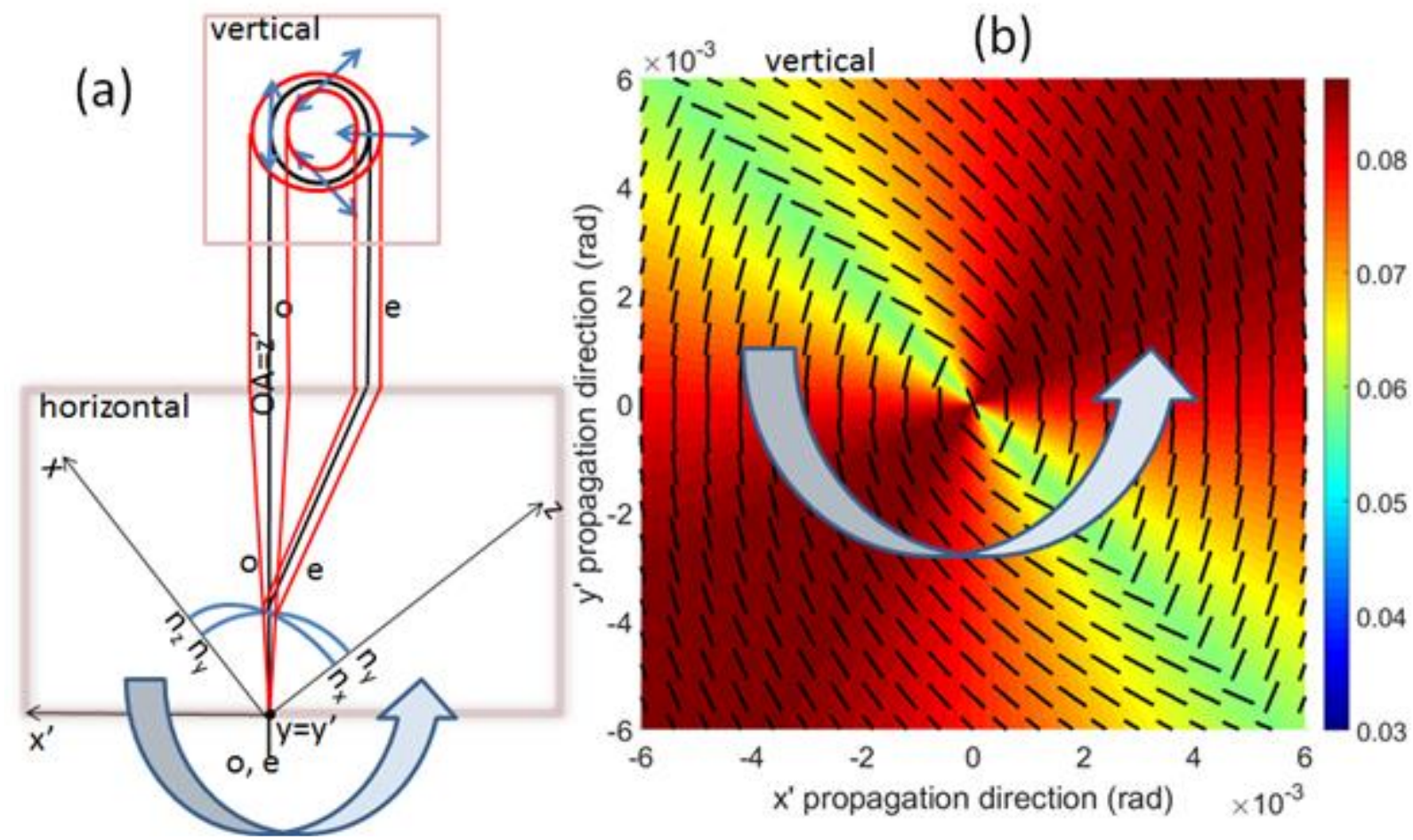

Fig. 2 (a) Crystal orientation and conical diffraction circle; (b) Fluorescence conoscopy of the highest emission cross-section mode. The color scale is the fluorescence intensity and the black lines are the mode polarization.

The principle of the experiment can be qualitatively explained with Fig. 2. In Fig. 2 (a) we can see the wellknown conical diffraction circle obtained if all the polarizations are contained in the incident Gaussian beam. However this is generally not the case if the beam is generated by the biaxial laser crystal itself (we could speak of "self-conical diffraction") with a propagation direction slightly different of the optical axis. The reason is that two modes exist for each propagation direction with a well-defined polarization and stimulated emission cross-section. Lasing is expected to select the mode with the highest emission cross-section. This mode is represented in Fig. 2 (b) as a result of a calculation verified by a separate fluorescence experiment [25]. Once the polarization has been selected in Fig. 2 (b) and going back to the circle in Fig. 2 (a) we can make the link between the expected crescent laser mode centered on that polarization and the propagation direction. This qualitative explanation is supported by a full calculation detailed in the final part of the paper. Different angular orientations of the laser crystal recorded with the CCD2 camera are reported in Fig. 3. The first remarkable set of orientations is marked with vertical black crescents, it is horizontal, it corresponds to 


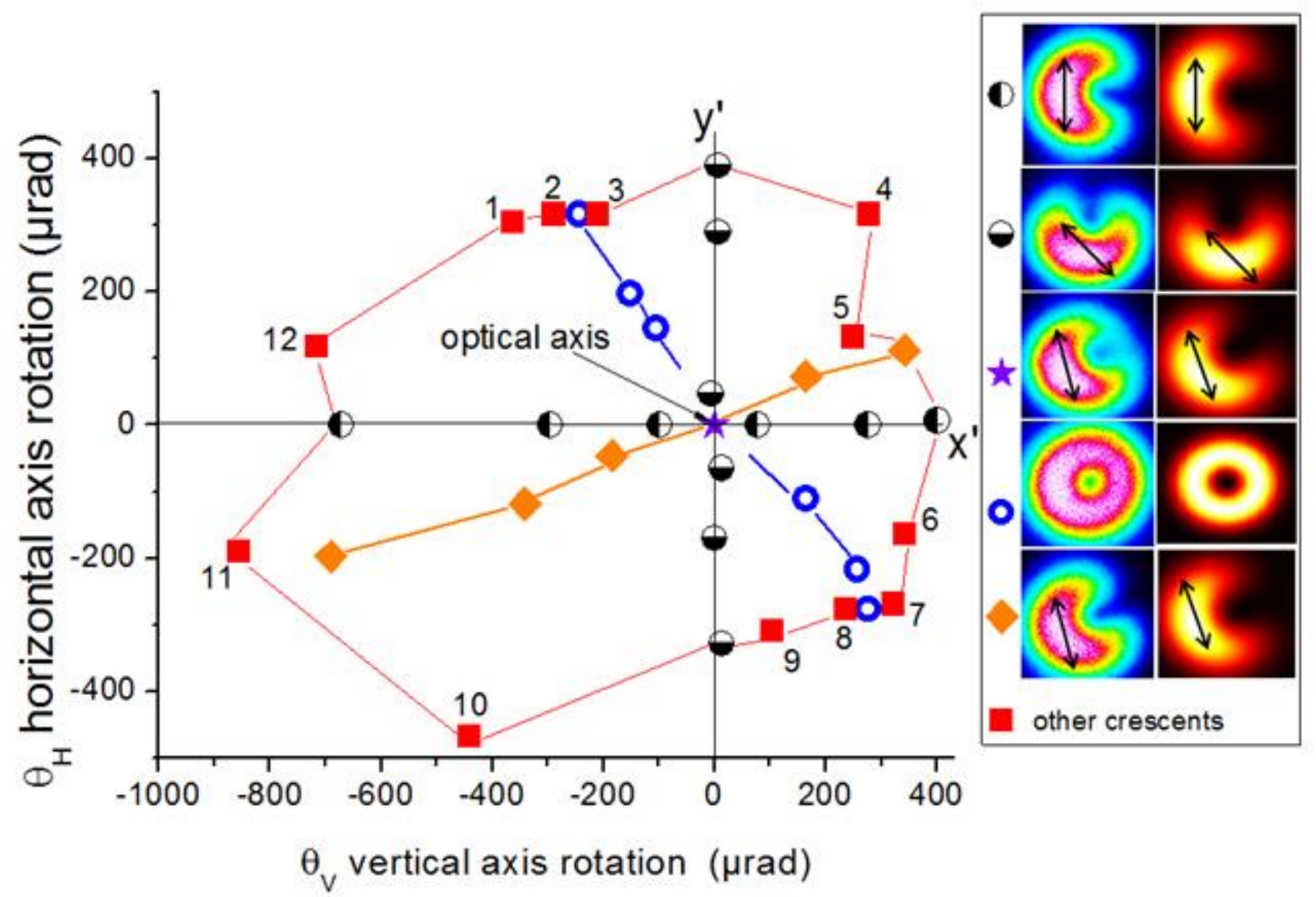

Fig. 3 Angular orientations explored: the positions of the symbols denote different adjustments of the optical axis away from the laser cavity axis. The six symbol families denote six kinds of crescent modes represented in the onset. Onset: remarkable laser modes obtained experimentally (left column) and theoretically (right column).

the experimental laser mode on the plane output mirror (visualized with the CCD3 camera) which is represented in the onset in Fig. 3 (left picture). So on the plane mirror the mode is crescent shape while it is Gaussian on the concave mirror. The measured polarization of the output laser beam (measured from the $1068 \mathrm{~nm}$ leaks through the concave mirror) is vertical with some mixing of unpolarised light. Going back to Fig. 2 (b) we attribute the horizontal locus to propagation directions in the $x-z$ principal plane and this is in agreement with the corresponding calculated near field mode (onset in Fig. 3, right column). The second remarkable set of angular orientations is marked with horizontal black crescents in Fig. 3 and also represented by such experimental crescents in the onset of Fig. 3 (left picture). The measured polarization of the laser beam is close to $135^{\circ}$ by respect with the $x^{\prime}$ axis. Going back to Fig. 2 (b) we attribute this vertical locus to propagation directions perpendicular to the $x-z$ principal plane, which is confirmed by the calculation (onset in Fig. 3 right column). The intersection of these two horizontal and vertical locus of angular positions is mandatory the optical axis. This is confirmed by calculation (star in Fig. 3). Let us notice 
that the measured polarization is not vertical but makes $110^{\circ}$ by respect to $x^{\prime}$ axis: this is the signature of the monoclinic symmetry of the crystal as we will explained in more detail below.

Let us go now to the orientations represented in Fig. 3 by the red squares and labelled "other crescents". Their experimental and theoretical near field laser modes are represented in Fig. 4 with a good agreement between experiment and theory. Two couples of points are of particular interest: $2 / 3$ and $7 / 8$. The two orientations in each couple are separated by a small horizontal angle: 80 and $86 \mu$ rad respectively (half of these values for the internal angles taking into account that the average refractive index is closed to 2). Despite the smallness of the separation, a drastic and spectacular transition is observed in the crescent mode (Fig. 4 pictures $2 / 3$ and $7 / 8$ ) on the conical refraction circle. This is the signature of an abrupt change $\left(90^{\circ}\right)$ in the polarization of the laser mode which stresses the following mechanism. First, a genuine optical lever arm is realized by angular geometrical magnification: $80 \mu \mathrm{rad}$ between the 2 and 3 horizontal positions lead to $10^{\circ}$ rotation around the optical axis in the transverse vertical plane, (i. e. X 2200 magnification). Second: the laser has a threshold behavior relaying the lever for a full mode selection. The $2 \rightarrow 3$ and $7 \rightarrow 8$ mode switching correspond to the abrupt change of the polarization of the highest emission cross-section mode visualized in the dark blue area in the fluorescence conoscopy picture Fig. 2 (b) (in this area the highest and lowest mode fluorescences have equal intensity [25]). Moving carefully the horizontal and vertical angle actuators in the crystal orientation region of abrupt crescent changes, it is possible to stabilize full circles (blue circles in Fig. 3). The set of these circles is not vertical: this is the signature of the monoclinic symmetry. The propagation directions correspond to a polarization singularity: abrupt change [1] with no defined polarization of the lasing mode. The $1068 \mathrm{~nm}$ Gaussian leaks trough the concave mirror were clearly found un-polarized. This measure was performed with a thermal power-meter with a slow response time, but we expect that the instantaneous polarization changes in time in a random manner as it is the case for beams originating from an isotropic laser medium without optical component inside the cavity to select the polarization. On the other hand the laser beam imaged by the CCD3 camera has a regular variation of its polarization around the ring as it is expected when the conical refraction is obtained with an input focused beam either un-polarized or circularly polarized (Fig. 2 (a)).

Finally we have explored other crystal orientations of interest: the orientations leading to the laser modes with the highest intensity (mW, measured with a power-meter). This set is represented in Fig. 3 by the orange diamond shapes. The mode polarization is closed to $103^{\circ}$ by respect to the horizontal axis. Let us notice that this diamond set is not horizontal and the polarization not vertical: this is another signature of the monoclinic symmetry.

The maximum output power measured at the exit of the plane output mirror was $44 \mathrm{~mW}$ for $480 \mathrm{~mW}$ pumping. The efficiency seems modest but we have to keep in mind that this laser was built for a fundamental study and no optimization was performed. For example the pump waist was enlarged by the 
concave mirror acting as a divergent lens which was not further compensated. The laser power versus polarization in another conical diffraction laser was studied in ref. [19].
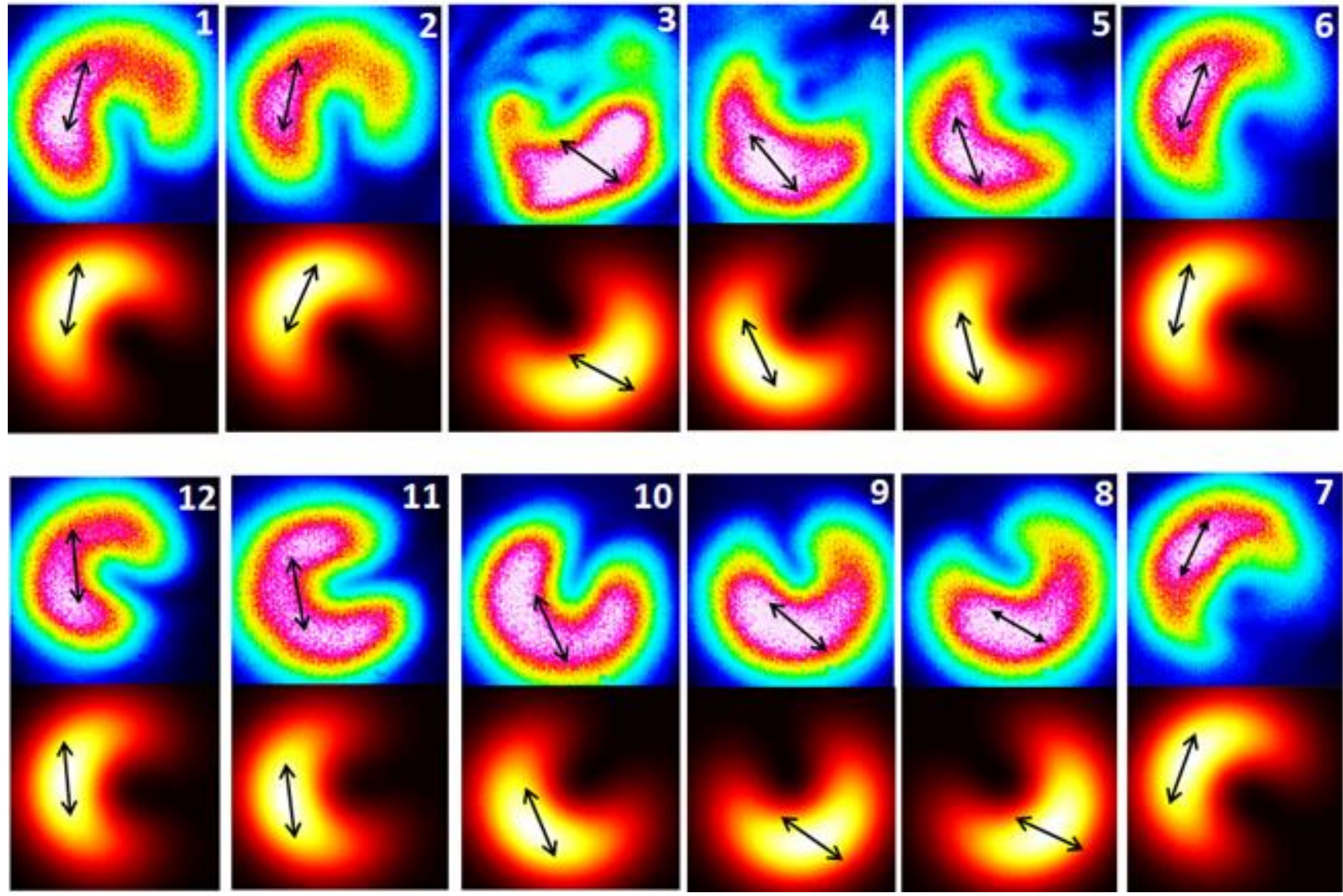

Fig. 4 Experimental (upper lines) and theoretical (lower lines) crescent modes obtained for the angular crystal orientations numbered if Fig. 3.

\section{Theoretical modelling}

We have modeled the off-optical axis conical refraction laser on the hypothesis that in a given propagation direction (beam central direction) the mode polarized with the highest stimulated emission cross-section will be established in the cavity and will be launched through the crystal. More precisely the beam propagating forward from the concave mirror towards the plane one is Gaussian when entering in the crystal, its central direction incident in the crystal being the z" axis whose coordinates in the (x'y'z') frame are obtained by the two $\theta_{v}$ and $\theta_{H}$ rotations making the $\left(x^{\prime \prime} y^{\prime \prime} z\right) \rightarrow\left(x^{\prime} y^{\prime} z^{\prime}\right)$ transfer:

$$
\left[\begin{array}{ccc}
1 & 0 & 0 \\
0 & \cos \left(\theta_{\mathrm{V}}\right) & -\sin \left(\theta_{\mathrm{V}}\right) \\
0 & \sin \left(\theta_{\mathrm{V}}\right) & \cos \left(\theta_{\mathrm{V}}\right)
\end{array}\right]\left[\begin{array}{ccc}
1 & 0 & 0 \\
0 & \cos \left(\theta_{\mathrm{H}}\right) & -\sin \left(\theta_{\mathrm{H}}\right) \\
0 & \sin \left(\theta_{\mathrm{H}}\right) & \cos \left(\theta_{\mathrm{H}}\right)
\end{array}\right]
$$


Once we are installed in the ( $\left.x^{\prime} y^{\prime} z^{\prime}\right)$ frame linked to the crystal thanks to the two previous rotations applied to any 3D-vectors (wave-vector and polarization), we can exploit the model performed in ref. [19] (with notations imported from [25)) and at the end go back to the ( $x^{\prime \prime} y^{\prime \prime} z^{\prime \prime}$ ) frame.

In particular we can obtain the transverse components of the two eigen-modes in the central incident direction:

$$
\left[\frac{1}{2 \delta_{22}-\delta_{11} \pm \Delta^{1 / 2}}\right]_{x 1 x 2}
$$

and we can select the mode with the highest emission cross-section corresponding to the highest imaginary part of the complex wave-number (represented in see Fig. 2 (b)):

$$
k_{ \pm}=\omega\left[\frac{\mu_{0}}{2 \underline{\epsilon}_{33}}\left\{\left(\delta_{11}+\delta_{22}\right) \pm \Delta^{1 / 2}\right\}\right]^{1 / 2}
$$

Another step requires the 2D-Fourier transform of the incident Gaussian beam. For that purpose, given the characteristics of the cavity (length: $4.7 \mathrm{~cm}$, concave mirror radius curvature: $5 \mathrm{~cm}$, distance plane mirrorcrystal: $2.1 \mathrm{~cm}$ ) and using the ABCD law for a round-trip [26] we determine the beam waist to be $70 \mu \mathrm{m}$.

The last important KGW:Nd feature is that this crystal is monoclinic and centrosymmetric. Its two-fold $\mathbf{b}$ crystallographic axis coincides with the $\mathbf{N p = x}(=\mathbf{b})$ principal axis of the dielectric frame. The imaginary part of the susceptibility tensor $\varepsilon=\varepsilon_{0}\left(\epsilon^{\prime}+\mathrm{i} \epsilon^{\prime \prime}\right)$ is diagonal in a $(\tilde{\mathrm{x}}, \tilde{\mathrm{y}}, \tilde{\mathrm{z}})$ frame deduced by a $\theta_{0}$-rotation of the $(\mathrm{x}, \mathrm{y}$, z) frame around the $\mathbf{x}(=\mathbf{b})$ axis as it is allowed by the monoclinic symmetry. This rotation means that generally absorption and fluorescence extrema do not coincide with the three principal $\mathrm{x}, \mathrm{y}, \mathrm{z}$ axes. A nondiagonal term $\varepsilon_{\mathrm{yz}}^{\prime \prime}$ appears if we express $\varepsilon^{\prime \prime}$ in the (xyz) frame from its diagonal expression in the $(\widetilde{\mathrm{x}}, \tilde{\mathrm{y}}, \tilde{\mathrm{z}})$ frame:

$$
\left[\begin{array}{ccc}
\varepsilon_{\mathrm{xx}}^{\prime \prime} & 0 & 0 \\
0 & \varepsilon_{\mathrm{yy}}^{\prime \prime} & \varepsilon_{\mathrm{yz}}^{\prime \prime} \\
0 & \varepsilon_{\mathrm{yz}}^{\prime \prime} & \varepsilon_{\mathrm{zz}}^{\prime \prime}
\end{array}\right]=\left[\begin{array}{ccc}
1 & 0 & 0 \\
0 & \cos \left(\theta_{0}\right) & \sin \left(\theta_{0}\right) \\
0 & -\sin \left(\theta_{0}\right) & \cos \left(\theta_{0}\right)
\end{array}\right] X\left[\begin{array}{ccc}
\varepsilon_{\tilde{\mathrm{x}} \tilde{\mathrm{x}}}^{\prime \prime} & 0 & 0 \\
0 & \varepsilon_{\tilde{\mathrm{y}} \tilde{\mathrm{y}}}^{\prime \prime} & 0 \\
0 & 0 & \varepsilon_{\tilde{\mathrm{z}} \tilde{\mathrm{z}}}^{\prime \prime}
\end{array}\right] X\left[\begin{array}{ccc}
1 & 0 & 0 \\
0 & \cos \left(\theta_{0}\right) & -\sin \left(\theta_{0}\right) \\
0 & \sin \left(\theta_{0}\right) & \cos \left(\theta_{0}\right)
\end{array}\right]
$$

Inspection of $\varepsilon_{\mathrm{ij}}^{\prime \prime}$ reveals that the $\theta_{0}$ angle can be obtained from the formula:

$$
\operatorname{tg}\left(2 \theta_{0}\right)=\frac{2 \varepsilon_{y z}^{\prime \prime}}{\varepsilon_{z z}^{\prime \prime}-\varepsilon_{y y}^{\prime \prime}}
$$

Such a rotation was already reported for KGW:Nd [25] and it was in the past encountered in the monoclinic $\mathrm{YCa}_{4} \mathrm{O}\left(\mathrm{BO}_{3}\right)_{3}: \mathrm{Nd}[27]$ and in other recent examples cited in [28].

The calculation procedure is: reading the crystal angular orientations in Fig. 3, launching through the crystal the Gaussian beam mode with the highest emission cross-section and calculating the near field pattern on the output plane mirror according to [19] and Equ. (1). We obtained reasonable agreement with 
the experimental patterns as shown in Fig. 3 (onset) and in Fig. 4. For that, we have adjusted two parameters. The first one is the population inversion, adjusted around $1.510^{-3}$ to get $5 \%$ amplification for a beam round trip, corresponding to the transmission of the plane output coupler. The second parameter is the $\theta_{0}$-angle (Equ. 5) adjusted to $27^{\circ}$ in order to describe simultaneously the mode switching visualized in Fig. 4 pictures $2 / 3$ and $7 / 8$. The predicted directions of singularity of this switching is $132^{\circ}$ with respect to the $\mathrm{x}^{\prime}$ horizontal axis, which is close to the blue circles positions in Fig. 3. The full circles are of course obtained launching a non-polarized beam.

More, the agreement between the theoretical and experimental eigen-mode angle polarization is also reasonably good for all the studied crystal orientations, as it is visualized by the black arrows in Fig. 3 (onset) and Fig. 4. We have to add that due to the weak population inversion the two eigen-modes in any direction are very close to be linearly polarized and, if $\psi$ is the propagation direction angle by respect to the $\mathrm{x}^{\prime}$ axis, their polarization angles are $\psi / 2$ and $\psi / 2+90^{\circ}$ as it is well-known in optics of transparent crystals. In particular the model predicts that the two orthogonal eigen-modes involved in the blue circles singularity have $66^{\circ}$ and $156^{\circ}$ angle by respect to the $x^{\prime}$ axis, and that the eigen-mode in the optical axis propagation direction and in directions with the highest laser power has $111^{\circ}$.

\section{Conclusion}

In conclusion, we based our optical study on a stimulated emission-active medium, the monoclinic $\mathrm{KGd}\left(\mathrm{WO}_{4}\right)_{2}$ :Nd (KGW:Nd) biaxial crystal cut perpendicular to the optical axis and verified by conoscopy. Thanks to controlling the off-optical axis propagation, we exhibited remarkable crescent laser modes and mode switching. We revealed for the first time that some optical singularities in addition to the optical axis leads to un-polarized lasing: the directions of abrupt change of the polarization of the mode with the highest emission cross-section. The key explanation is that lasing occurs in the mode with the lowest threshold, the later one depending drastically on the crystal orientation. In other words a genuine optical lever arm is realized between the propagation directions variations and the transverse directions with an angular geometrical magnification above 2000. A model taking into account a two-axes crystal rotation and describing the conical diffraction behavior is provided to support fully the experimental data. As a

supplementary effect the rotation around the two-fold $\mathbf{b}$ crystallographic axis of the frame which makes diagonal the imaginary part of the dielectric susceptibility tensor is exhibited all along the study.

Acknowledgment. We thank Y. Guillin, J.-F. Sivignon and J.-M. Ingargiola for their technical assistance. 


\section{References}

1. M. Berry, Nature, 403 (6), 21, 2000.

2. A. J. Danner, T. Tyc, U. Leonhardt, “Nature Photonics, 5, 357, 2011.

3. C. V. Raman, V. S. Rajagopalan and T. M. K. Nidungadi, Proc. Ind. Acad. Sci. A, vol. 14, 221, 1941.

4. A. J. Shell and N. Bloembergen, J. Opt. Soc. Am. 68 (8), 1093, 1978.

5. A. M. Belskii and M. A. Stepanov, Opt. Comm. 167, 1-5 (1999).

6. M. V. Berry, J. Opt. A: Pure Appl. Opt. 6, 289-300 (2004).

7. A. Turpin, Y. Loiko, T. Kalkandjiev, J. Mompart, Opt. Lett. 37, 4197-4199 (2012).

8. D. P. O’Dwyer, K. E. Ballantine, C. F. Phelan, J. G. Lunney, J. F. Donegan, Opt. Exp. 20, 21119-21121 (2012).

9. S. Rosen, G. Y. Sirat, H. Ilan, and A. J. Agranat, Opt. Exp. 21, 10133-10138 (2013).

10. J. Caron, C. Fallet, J.-Y. Tinevez, L. Moisan, L. P. (Ori) Braitbart, G. Y. Sirat, and S. L. Shorte, Cell Adhesion \& Migration, 8:5, 430-439 (2015).

11. C. F. Phelan, R. J. Winfield, D. P. O’Dwyer, Y. P. Rakovich, J. F. Donegan, and J. G. Lunney, Opt. Commun. 284, 35713574 (2011).

12. A. Peinado, A. Turpin, A. Lizana, E. Fernández, J.i Mompart, and J. Campos, Opt. Lett. 38, 4100-4103 (2013).

13. S. D Grant, S. Reynolds and A. Abdolvand, J. Opt. 18, 025609 (2016).

14. J. Hellstrom, H. Henricsson, V. Pasiskevicius, U. Bunting, D. Haussmann, Opt. Lett. 32, 2783-2785 (2007).

15. K. G. Wilcox, A. Abdolvand, T. K. Kalkandjiev, E. U. Rafailov, Appl. Phys. B Lasers and Optics 99, 619-622 (2010).

16. A. Abdolvand, K. G. Wilcox, T. K. Kalkandjiev, E. U. Rafailov, Opt. Exp. 18, 2753-2759 (2010).

17. Y. V. Loiko, G. S. Sokolovskii, D. Carnegie, A. Turpin, J. Mompart, E. Rafailov, Proc. SPIE 8960, 89601Q (2014).

18. Romain Cattoor, Inka Manek-Hönninger, Daniel Rytz, Lionel Canioni, and Marc Eichhorn, Opt. Lett. 39, 64076410 (2014).

19. A. Brenier, Appl. Phys.B: Lasers and Optics, 122, 237, (2016).

20. S. Pancharatnam, Proc. Ind. Acad. Sci. 42 A, 86-109 and 235-248 (1955).

21. A. Brenier, J. Opt. 17, 075603 (2015).

22. J. Gerardin, A. Lakhtakia, Optik 112, 493-495 (2001).

23. Tom G. Mackay, J. Nanophot. 8, 083993 (2014).

24. T. G. Mackay and A. Lakhtakia, J. Opt. A: Pure Appl. Opt. 5, 91, (2003).

25. A. Brenier, Las. Phys. Lett. 11, 115819, (2014).

26. Yariv A 1991 Optical Electronics 4th edn (USA: Saunders College Publishing).

27. Y. Petit, B. Boulanger, P. Segond, C. Félix, B. Ménaert, J. Zaccaro , G. Aka, Opt. Exp. 16 (11) 7997-8002 (2008).

28. L. Cabaret, J. Robert, K. Lebbou, A. Brenier, H. Cabane, Optical Materials, 62, 597 (2016). 


\section{References with titles}

1. M. Berry, "Making waves in physics”, Nature, 403 (6), 21, 2000.

2. A. J. Danner, T. Tyc, U. Leonhardt, “Controlling birefringence in dielectrics”, Nature Photonics, 5, 357, 2011.

3. C. V. Raman, V. S. Rajagopalan and T. M. K. Nidungadi, “Conical refraction in naphthalene crystals”, Proc. Ind. Acad. Sci. A, vol. 14, 221, 1941.

4. A. J. Shell and N. Bloembergen, "Laser studies of internal conical diffraction. I. Quantitative comparison of experimental and theoretical conical intensity distribution in aragonite”, J. Opt. Soc. Am. 68 (8), 1093, 1978.

5. A. M. Belskii and M. A. Stepanov, "Internal conical refraction of coherent light beams”, Opt. Comm. 167, 1-5 (1999).

6. M. V. Berry, "Conical diffraction asymptotics: fine structure of Poggendorff rings and axial spikes”, J. Opt. A: Pure Appl. Opt. 6, 289-300 (2004).

7. A. Turpin, Y. Loiko, T. Kalkandjiev, J. Mompart, "Free-space optical polarization demultiplexing and multiplexing by means of conical refraction", Opt. Lett. 37, 4197-4199 (2012).

8. D. P. O’Dwyer, K. E. Ballantine, C. F. Phelan, J. G. Lunney, J. F. Donegan, “Optical trapping using cascade conical refraction of light", Opt. Exp. 20, 21119-21121 (2012).

9. S. Rosen, G. Y. Sirat, H. Ilan, and A. J. Agranat, "A sub wavelength localisation scheme in optical imaging using conical refraction", Opt. Exp. 21, 10133-10138 (2013).

10. J. Caron, C. Fallet, J.-Y. Tinevez, L. Moisan, L. P. (Ori) Braitbart, G. Y. Sirat, and S. L. Shorte, “Conical diffraction illumination opens the way for low phototoxicity super-resolution imaging”, Cell Adhesion \& Migration, 8:5, 430439 (2015).

11. C. F. Phelan, R. J. Winfield, D. P. O’Dwyer, Y. P. Rakovich, J. F. Donegan, and J. G. Lunney, Opt. Commun. 284, 35713574 (2011).

12. A. Peinado, A. Turpin, A. Lizana, E. Fernández, J.i Mompart, and J. Campos, "Conical refraction as a tool for polarization metrology," Opt. Lett. 38, 4100-4103 (2013).

13. S. D Grant, S. Reynolds and A. Abdolvand, "Optical sensing of polarization using conical diffraction phenomenon," J. Opt. 18, 025609 (2016).

14. J. Hellstrom, H. Henricsson, V. Pasiskevicius, U. Bunting, D. Haussmann, "Polarization-tunable Yb:KGW laser based on internal conical refraction", Opt. Lett. 32, 2783-2785 (2007).

15. K. G. Wilcox, A. Abdolvand, T. K. Kalkandjiev, E. U. Rafailov, "Laser with simultaneous Gaussian and conical refraction outputs", Appl. Phys. B Lasers and Optics 99, 619-622 (2010).

16. A. Abdolvand, K. G. Wilcox, T. K. Kalkandjiev, E. U. Rafailov, “Conical refraction Nd:KGd($\left(\mathrm{WO}_{4}\right)_{2}$ laser”, Opt. Exp. 18, 2753-2759 (2010).

17. Y. V. Loiko, G. S. Sokolovskii, D. Carnegie, A. Turpin, J. Mompart, E. Rafailov, "Laser beams with conical refraction patterns", Proc. SPIE 8960, 89601Q (2014).

18. Romain Cattoor, Inka Manek-Hönninger, Daniel Rytz, Lionel Canioni, and Marc Eichhorn, "Laser action along and near the optic axis of a holmium-doped KY(WO4)2 crystal," Opt. Lett. 39, 6407-6410 (2014). 
19. A. Brenier, "Lasing with conical diffraction feature in the $\mathrm{KGd}\left(\mathrm{WO}_{4}\right)_{2}: \mathrm{Nd}$ biaxial crystal”, Appl. Phys.B: Lasers and Optics, 122, 237, (2016).

20. S. Pancharatnam, "The propagation of light in absorbing biaxial crystals", Proc. Ind. Acad. Sci. 42 A, 86-109 and 235-248 (1955).

21. A. Brenier, "Voigt wave investigation in the $\mathrm{KGd}\left(\mathrm{WO}_{4}\right)_{2}$ :Nd biaxial laser crystal", J. Opt. 17, 075603 (2015).

22. J. Gerardin, A. Lakhtakia, "Conditions for Voigt wave propagation in linear, homogenous, dielectric medium"s, Optik 112, 493-495 (2001).

23. Tom G. Mackay, J. Nanophot. "On the sensitivity of directions that support Voigt wave propagation in infiltrated biaxial dielectric materials", 8, 083993 (2014).

24. T. G. Mackay and A. Lakhtakia, "Voigt wave propagation in biaxial composite materials”, J. Opt. A: Pure Appl. Opt. 5, 91, (2003).

25. A. Brenier, "Polarization properties of lasing near an optical axis in the biaxial crystal”, Las. Phys. Lett. 11, 115819, (2014).

26. Yariv A 1991 Optical Electronics 4th edn (USA: Saunders College Publishing).

27. Y. Petit, B. Boulanger , P. Segond, C. Félix, B. Ménaert, J. Zaccaro , G. Aka, “Absorption and fluorescence anisotropies of monoclinic crystals : the case of Nd :YCOB", Opt. Exp. 16 (11) 7997-8002 (2008).

28. L. Cabaret, J. Robert, K. Lebbou, A. Brenier, H. Cabane, "Growth, spectroscopy and lasing of the Yb-doped monoclinic $\mathrm{Gd}_{2} \mathrm{SiO}_{5}$ in the prospect of hydrogen laser cooling with Lyman- $\alpha$ radiation", Optical Materials, 62, 597 (2016). 